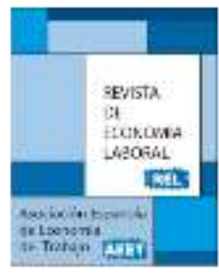

\title{
LOS RIESGOS DE AUTOMATIZACIÓN Y DESLOCALIZACIÓN DE LOS TRABAJADORES AUTÓNOMOS ${ }^{1}$
}

\author{
M.Á. Malo ${ }^{2 *}$ y B. Cueto ** \\ * Universidad de Salamanca \\ ** Universidad de Oviedo
}

Recibido Septiembre 2019; Aceptado Diciembre 2019

\section{Resumen}

En este artículo, se estiman los riesgos de automatización y deslocalización de los trabajadores autónomos en España, para el periodo 2011-2018. La investigación parte de resultados previos que muestran que dichos riesgos son mucho menores para los autónomos que para los asalariados, en especial el de automatización. Nuestro análisis muestra que estas diferencias tienen poco que ver con que los autónomos tengan diferentes características, si bien estas parecen tener algo de importancia en el caso del riesgo de deslocalización. Los resultados apuntan a la necesidad de utilizar metodologías diferentes de las convencionales para estimar dichos riesgos, pues parece haber diferencias en el contenido de las mismas ocupaciones cuando las desempeñan autónomos en lugar de asalariados.

Palabras clave: autoempleo; globalización; cambio técnico; ocupación.

Clasificación JEL: J21; J23; L26

\section{Abstract}

In this article, we estimate the risks of automation and offshorability for selfemployed workers in Spain, for the period 2011-2018. The starting point is a previous result showing that these risks are much lower for self-employed people than for wage earners. Our analysis shows that these differences seems unrelated to different characteristics of selfemployed people respect to employees in the case of the automation risks, while differences in characteristics may play some role for the offshorability risk. The results suggest moving towards new methodologies to estimate these risks, because of the possibility that the tasks of the same occupation differ for self-employed workers respect to wage earners.

Key words: self-employment; globalization; technical change; occupation.

JEL Classification: J21; J23; L26

\footnotetext{
1 Miguel Ángel Malo agradece la financiación recibida del proyecto de investigación CSO2014-599927-R del Ministerio de Economía y Competitividad, y del proyecto de investigación SA072U16 de la Junta de Castilla y León. Begoña Cueto agradece la financiación recibida del proyecto de investigación PAPI-19-GR-2014-0076 de la Universidad de Oviedo.

${ }^{2}$ Correos de contacto: malo@usal.es y bcueto@uniovi.es
}

(C) Revista de Economía Laboral 


\section{Introducción}

El objetivo de este artículo consiste en estimar los riesgos de automatización y deslocalización de los trabajadores autónomos utilizando datos de España. La estimación de dichos riesgos en relación con el empleo agregado o con los asalariados es un tema al que la investigación reciente ha dedicado grandes esfuerzos en el pasado reciente (Frey y Osborne, 2017; Arntz et al., 2017; Nedelkoska y Quintini, 2018; Pouliakas, 2018). No obstante, apenas se ha discutido ni en la literatura académica ni en el debate político cuáles pueden ser esos riesgos para el caso del trabajo por cuenta propia.

El debate social ha estado muy centrado en las eventuales pérdidas de empleo asociadas con la automatización y la digitalización (Hidalgo, 2018). Para el empleo total, se cuentan con estimaciones que van desde un riesgo de pérdida o transformación profunda de prácticamente la mitad de todo el empleo (Frey y Osborne, 2017) hasta otras que lo sitúan entre el 10 y el 20 por ciento (Arntz et al, 2017; Nedelkoska y Quintini, 2018). Además, la estimación de estos riesgos no tiene en cuenta el potencial impacto positivo (directo e indirecto) de los cambios mencionados sobre el empleo total, por lo que la estimación de estos riesgos tiene la lógica de intentar anticipar qué puede ir mal en relación con el cambio tecnológico y tomar medidas, en su caso, que puedan paliar esos potenciales efectos negativos sobre el empleo.

Cabe destacar que el posible impacto de los riesgos de automatización y de deslocalización no tienen por qué afectar al empleo de igual manera ni distribuirse de manera semejante entre los trabajadores. Resulta frecuente en el debate social escuchar que la globalización lleva a deslocalizar las tareas más rutinarias y de menor cualificación hacia otros países, por lo que las potenciales pérdidas de empleo serían parecidas a buena parte de las que se esperan de la automatización (en cuanto a la conexión de esta con la rutinización de tareas). Ahora bien, hay que tener en cuenta que resulta muy difícil deslocalizar ocupaciones que, aunque tengan un componente rutinario fuerte o estén desempeñadas por personal con baja cualificación, también necesiten una importante interacción social o estén fuertemente vinculados a la economía local. Por tanto, esta presunción habitual dista mucho de ser correcta, tal como ha mostrado la investigación previa sobre deslocalización y empleo (Blinder, 2009; Blinder y Krueger, 2013).

La literatura académica previa se ha limitado a analizar el impacto de los riesgos de automatización y globalización sobre el empleo total o, si acaso, sobre el empleo asalariado, pero apenas se ha 
profundizado en si cabe esperar algún tipo de impacto diferenciado sobre el trabajo por cuenta propia en comparación con el asalariado. Hay evidencia empírica que apunta que la creciente automatización aumentaría las transiciones del empleo asalariado hacia el autoempleo en las ocupaciones con bajo riesgo de automatización, debido a que la transformación digital estaría creando nuevas oportunidades en ese tipo de ocupaciones más fáciles de explotar desde una posición de autoempleado que desde una de asalariado (Sorgner, 2017). En cuanto al riesgo de deslocalización del empleo por cuenta propia, hasta donde sabemos, no existe literatura previa más allá de la evidencia aportada por Anderson y Gascon (2008), quienes encuentran que los autónomos son quienes más riesgo subjetivo declaran de perder su empleo en el contexto de una creciente globalización. Por otro lado, disponemos de una estimación agregada para el caso español que muestra un riesgo de automatización y deslocalización de los autoempleados y los empresarios en comparación con los asalariados (Malo y Cueto, 2019).

Este trabajo pretende profundizar en el resultado anterior, proporcionando una evidencia empírica más detallada del caso español sobre dicha diferencia en los riesgos de automatización y deslocalización. Dada la gran escasez de literatura previa sobre cómo afectan este tipo de riesgos al empleo autónomo, los resultados de este trabajo (relacionados con las características y limitaciones de las bases de datos existentes) vienen a llenar una laguna en esta línea de investigación y apuntan a diferentes caminos para su desarrollo en el futuro inmediato. Como toda la literatura sobre los riesgos para el empleo de la automatización y la deslocalización, nuestro trabajo es descriptivo. Así pues, los resultados deben entenderse como relaciones de interés entre determinadas variables y como la búsqueda de esas relaciones a un nivel agregado. Dada la carencia de información sobre el tema en España, consideramos que los resultados tienen interés para anticipar algunas tendencias importantes e informar, en su caso, las políticas preventivas teniendo en cuenta las diferencias entre empleo por cuenta propia y ajena.

Los datos de esta investigación utilizan indicadores previamente desarrollados tanto en la investigación sobre automatización (Frey y Osborne, 2017) como en la de globalización y deslocalización del empleo (Blinder y Krueger, 2013). Uniremos estos indicadores a los micro-datos de la Encuesta de Población Activa (EPA) para el periodo 2011 a 2018. Así pues, los resultados deberán considerarse como un promedio de dichos años. En pro de un análisis más rico, hemos utilizado la versión de la EPA con una desagregación de tres dígitos de la actual clasificación nacional de ocupaciones. En cuanto a otras variables utilizadas hemos 
preferido centrarnos en variables que no cambian a lo largo del tiempo y que, a la vez, en la literatura previa muestran una diferenciación importante en los riesgos considerados, como ocurre con el sexo y el nivel educativo (Malo y Cueto, 2019).

La estructura del artículo es como sigue. En la siguiente sección, se discute la importancia de los nuevos riesgos del empleo asociados al cambio tecnológico y la globalización, en cuanto a su impacto sobre el empleo total e introduciendo las diferencias que podrían afectar al empleo por cuenta propia. A continuación, se discute la metodología utilizada para aplicar los indicadores de riesgo de automatización y deslocalización a los datos españoles de la EPA. El análisis empírico consiste en diferentes explotaciones descriptivas para mostrar las peculiaridades del empleo autónomo respecto del empleo asalariado. Una sección de discusión y conclusiones cierra el artículo, proponiendo algunas líneas de investigación para el futuro inmediato.

\section{Los nuevos riesgos del empleo y el empleo autónomo}

El futuro del trabajo es una de las preocupaciones recurrentes del debate social y académico sobre el mercado de trabajo durante los últimos años. Por ejemplo, tanto la OIT como la OCDE han dedicado ingentes esfuerzos para llamar a la reflexión y a la elaboración de medidas ante los cambios que se avecinan en el mercado de trabajo (OIT, 2018; OCDE, 2019). Casi siempre, hablar del futuro del trabajo implica hablar bien de la creciente automatización (Frey y Osborne, 2017; Arntz et al., 2016), bien de la deslocalización causada por la globalización (Blinder and Krueger, 2013) o bien de las dos cuestiones a la vez, que se alimentarían mutuamente (Baldwin, 2019).

El impacto de la automatización sobre el empleo es posiblemente el mejor estudiado. La aproximación que ha tenido más repercusión ha consistido en la estimación del riesgo de automatización de las ocupaciones, vistas desde una perspectiva de conjunto o vistas como compuestas por haces de tareas. Con la primera perspectiva, Frey y Osborne (2017) estiman que un 47 por ciento de los empleos en EEUU tienen un riesgo elevado de automatización. Aplicando dichos riesgos a la estructura ocupacional española, Domenech et al. (2018) estiman que dicho riesgo elevado de automatización afectaría al 36 por ciento del empleo español (promedio de 2011 a 2016), mientras que Malo y Cueto (2019) estiman un 33,9 por ciento (promedio de 2011 a 2018). Utilizando 
la perspectiva del riesgo de automatización de las tareas que componen las ocupaciones Pouliakas (2018) y Nedelkoska y Quintini (2018) lo reducen al 14 por ciento para el caso de la Unión Europea y al 9 por ciento si se considera la OCDE (Arntz et al., 2016). Con la perspectiva de la automatización de las tareas, y a partir de los datos de Torrejón (2018), Malo y Cueto (2019) estiman que en España el 33,2por ciento del empleo tendría un riesgo elevado de automatización. ${ }^{3}$

Ahora bien, según Baldwin (2019), la conjunción de la automatización con la globalización (y el consecuente riesgo de deslocalización) puede dar un carácter único al impacto de la automatización sobre el empleo. Baldwin (2019) basa su argumentación en la existencia de "tele-migrantes" a través de plataformas digitales, si bien la globalización puede tener un impacto mucho más directo sobre el empleo a través del riesgo de deslocalización (Blinder y Krueger, 2013). En definitiva, conviene analizar conjuntamente los riesgos generados para el empleo tanto por la automatización como por la deslocalización para tener una perspectiva más amplia de las transformaciones a las que podría verse sometido el empleo en el futuro próximo (Malo y Cueto, 2019). Por otro lado, todos los trabajos anteriores se han centrado o en el empleo agregado sin detenerse a analizar el empleo por cuenta propia o bien solo ofrecen una desagregación sencilla entre empleo por cuenta propia y ajena sin entrar en más profundidades. En esta última línea, Malo y Cueto (2019) ofrecen el riesgo de automatización y deslocalización del empleo por cuenta propia, separando empleadores (empresarios y autónomos con asalariados) y autónomos sin asalariados. Sus estimaciones muestran un riesgo mucho menor tanto de automatización como de deslocalización para el trabajo por cuenta propia.

Los menores niveles de riesgo de automatización y de deslocalización entre los autónomos pueden deberse a varias razones. En primer lugar, es posible que los autónomos estén más concentrados en ocupaciones que tienen, por sí mismas y con independencia del tipo de relación de empleo, unas características que las protegen de dichos

\footnotetext{
${ }^{3}$ La distribución del riesgo de automatización está mucho más concentrada en Torrejón (2018) que en las otras estimaciones del riesgo, por lo que riesgo elevado no significa estar por encima de un riesgo de automatización del 70 por ciento, ya que, en sus estimaciones, no hay ninguna ocupación que tenga un riesgo por encima de dicho valor. En Malo y Cueto (2019), se considera que con la metodología de Torrejón (2018) un riesgo elevado de automatización comparable al de la metodología de Frey y Osborne (2017) es el umbral del 19 por ciento. Este problema de diferentes umbrales, y que la metodología de las tareas sólo se podía aplicar a datos españoles usando una desagregación a dos dígitos de la clasificación de ocupaciones, nos decantó a usar en el presente artículo sólo los riesgos de automatización estimados con la metodología de Frey y Osborne (2017).
} 
riesgos. En segundo lugar, podría suceder que el hecho de ser trabajadores autónomos les confiera algún tipo de diferencia crucial frente al hecho de ser asalariado en la misma ocupación. Esto podría ocurrir si la capacidad de tomar decisiones sobre su propio trabajo (organización, horario, motivación, etc.) les confiriera una flexibilidad, capacidad de adaptación o competitividad que hiciera sus puestos de trabajos menos susceptibles de automatización y/o deslocalización. Como veremos en el análisis empírico, no podemos explorar esta segunda razón debido a la metodología con la que se construyen los indicadores de riesgo de automatización y de deslocalización en las fuentes que utilizamos, y que consiste en una cierta estimación del riesgo para cada ocupación con independencia del tipo de relación de empleo que mantenga la persona. Esto nos lleva a tener que centrarnos en la primera cuestión, es decir, a centrar el análisis en la concentración relativa de autónomos y asalariados en determinadas ocupaciones.

Hay que señalar que la distribución de los autónomos entre sectores de actividad ha tenido una evolución cambiante a lo largo del pasado siglo y que, en el actual, posiblemente cambie más aún debido al impacto del cambio tecnológico en la organización del trabajo. La tendencia a lo largo del siglo XX consistió en una reducción del empleo autónomo dedicado a la agricultura y, en general, al sector primario, en consonancia con la transformación de las economías occidentales primero en economías industriales y, luego, en economías de servicios (Arum y Müller, 2009; Bögenhold, Klinglmair y Kandutsch, 2018). Fue precisamente la terciarización de las economías lo que impulsó el crecimiento del empleo por cuenta propia.

La regulación de las figuras de empleo ha tratado, en general, al empleo por cuenta propia como todo aquel que no es por cuenta ajena. En este sentido, la publicación del Estatuto del Trabajador Autónomo en 2007 supuso una gran innovación en la medida en que no había regulación del trabajador autónomo como tal (Cabeza Pereiro, 2008: 91).

La incertidumbre económica, el creciente proceso de "fisurización" del centro de trabajo (Weill, 2014) y el uso estratégico de la normativa laboral ha llevado a la aparición del fenómeno de los autónomos dependientes (Muehlberger, 2007; Malo, 2018), figura reconocida en el mencionado Estatuto como "trabajador económicamente dependiente TRADE”. Estos trabajadores están bajo la regulación jurídica del empleo autónomo (no son asalariados) pero mantienen una relación de dependencia respecto de su empresa cliente (una que les suministra todo su negocio o casi todo su negocio). De esta forma, la empresa estaría desplazando riesgos e incertidumbres hacia estos trabajadores como si 
éstos fueran una empresa, cuando lo único que sucede es que se explotan huecos de la normativa para disfrazar como autoempleo lo que es, en realidad, una relación laboral asalariada (dependiente). En Eurofound (2016: 24), se estima que, en Europa, los autónomos dependientes serían el 1 por ciento del empleo total y en torno al 13 de los autónomos sin asalariados, estando en España en un 0,5 por ciento del empleo total. No resulta descabellado pensar que si este fenómeno desvía incertidumbre del seno de la empresa hacia ese trabajador que pasa a ser autónomo, éstos estuvieran concentrándose en sectores donde la incertidumbre económica es mayor y, por tanto, la variabilidad de las ventas (y, por tanto, de ingresos) más elevada.

Más recientemente, los recientes cambios tecnológicos están detrás de la expansión del trabajo a través de plataformas en la llamada "gig economy", la cual está en gran medida unida al uso de la figura del trabajador autónomo. Dado el grado de dependencia de estos autónomos respecto de las plataformas para las que trabajan, existe una fuerte discusión jurídica en diferentes países sobre el estatus jurídico al que deben adscribirse (De Stefano, 2016; Rodríguez Fernández, 2019). Al igual que en el caso general de los falsos autónomos mencionado arriba, estos nuevos autoempleados concentrarían riesgos de variabilidad de actividad y de ingresos que, bajo una regulación laboral distinta, se gestionarían dentro de una relación laboral asalariada.

Cada país está reaccionando de una manera distinta al fenómeno de los autónomos dependientes. Por un lado, algunos autores reclaman una mejor diferenciación de las diferentes figuras de empleo, mientras que otros abogan por igualar los derechos sociales de todos los trabajadores sean éstos por cuenta propia o ajena (Rodríguez Fernández, 2019). España ha optado por crear la figura del trabajador autónomo dependiente (TRADE), que se situaría entre el autónomo y el asalariado, con acceso también a una protección intermedia (Malo, 2018).

Aunque el fenómeno del empleo a través de plataformas es creciente (Kässi y Lehdonvirta, 2018; Malo, 2018), su representación en relación con el stock total de empleo sigue siendo bastante reducido. De hecho, las estimaciones más recientes apuntan a que son un pequeño porcentaje del total: 0,5 por ciento del empleo total en EEUU, según Katz y Krueger (2016) y un 0,05 por ciento en la UE, según De Groen y Maselli (2016). Esto significa que se trata de un colectivo todavía difícil de captar a través de las fuentes estadísticas habituales, por lo que no cabe esperar que tenga un impacto relevante en nuestro análisis.

En cualquier caso, la expansión de estos tipos de trabajador autónomo no conllevaría con claridad ni mayores ni menores riesgos de 
automatización o deslocalización en comparación con los asalariados. Nótese que se trataría de desempeñar la misma ocupación tanto en el caso de ser asalariado como en el de ser un falso autónomo, por lo que, dada la forma de construcción de nuestros indicadores, tendrían el mismo riesgo de automatización y/o deslocalización.

\section{Análisis empírico}

\subsection{La medición de los riesgos de automatización y deslocalización}

La manera en que se miden los riesgos de automatización y deslocalización para el empleo se enfrenta a importantes desafíos prácticos que las diferentes aproximaciones han solucionado de formas distintas. Revisamos brevemente estas aproximaciones a continuación.

\subsubsection{Riesgo de automatización}

Quizá el trabajo más relevante para analizar el impacto del riesgo de automatización sobre el empleo es el publicado por Frey y Osborne en 2017, si bien una versión previa del mismo está disponible desde 2013. Los autores utilizan una perspectiva basada en las ocupaciones. A partir de entrevistas con expertos, identificaron los cuellos de botella en ingeniería a los que hacen frente los desarrolladores de aprendizaje automático y robótica móvil. Esta información se utilizó para definir el riesgo de automatización en 70 ocupaciones. Por otra parte, la clasificación ocupacional $\mathrm{O}$ *NET permitía disponer de datos sobre las tareas que componían cada una de las ocupaciones, que se clasificaban como potencialmente automatizables o no totalmente automatizables. La información básica de estas 70 ocupaciones analizadas por expertos sirvió para predecir la probabilidad de automatización de 702 ocupaciones disponibles en $\mathrm{O}$ *NET, estudiando así el impacto de la automatización sobre el mercado de trabajo en Estados Unidos. Los autores definieron tres niveles de riesgo: alto, si la probabilidad de automatización estaba entre 0.7 y 1 ; medio, si la probabilidad de automatización estaba entre 0.3 y 0.7 ; y bajo, si dicha probabilidad era menor de 0.3 . De acuerdo con sus estimaciones, un 47 por ciento de los empleados en Estados Unidos tenía un riesgo alto, un 19 un riesgo medio y un 33 un riesgo bajo.

La polémica suscitada por el cálculo de que casi la mitad del empleo estadounidense estaría bajo un riesgo elevado (superior al 70 por ciento) de automatización llevó a otros autores a proponer metodologías 
diferentes para medir este riesgo. A partir de los trabajos basados en la rutinización de las tareas (sobre todo, Autor et al., 2003), Arntz et al. (2016) construyeron indicadores de riesgo de automatización que se corresponderían básicamente con la rutinización de las tareas que componen cada ocupación. La información sobre la rutinización de las tareas se recoge mediante encuestas a trabajadores. Con esta metodología, el riesgo de automatización de una ocupación es la media del riesgo de automatización de cada tarea.

\subsubsection{Riesgo de deslocalización}

El riesgo de externalización o deslocalización se ha obtenido a partir de Blinder y Krueger (2013). Los autores utilizan una encuesta específica con información detallada sobre el tema y estiman tres indicadores distintos. Dos de ellos están basados en información subjetiva y el tercero recoge la opinión de un experto. El análisis de los autores concluye que este último es el más fiable, por lo que será el que se utilice en este trabajo.

Este indicador ofrece cinco valoraciones posibles del riesgo de deslocalización de una ocupación, desde no deslocalizable a deslocalizable con menor o ninguna dificultad. ${ }^{4}$ Con la encuesta original norteamericana, Blinder y Krueger (2013) estimaron que el 67.5 por ciento correspondía a ocupaciones no deslocalizables. Debido a esta gran concentración de casos y al bajo número en el resto, nosotros hemos optado por definir solo dos categorías: 0, no deslocalizable, y 1, deslocalizable. Este segundo grupo une todas las demás situaciones. Al transformar la información original basada en una clasificación de ocupaciones con 6 dígitos de desagregación en la que nosotros utilizamos en los datos españoles con solo 3 dígitos, tenemos algunos casos a los que se atribuyen dos o más riesgos de deslocalización. En estos casos hemos atribuido la media. Como resultado, tenemos algunas ocupaciones a 3 dígitos con riesgos entre cero y la unidad, es decir, tenemos tres categorías de riesgo: riesgo 0 , riesgo 1 o riesgo entre 0 y 1 . En cualquier caso, el 64,9 por ciento de nuestra muestra tiene un riesgo nulo de deslocalización, valor que se encuentra cercando al 67,5 por ciento de la encuesta norteamericana original. Para el máximo de riesgo de deslocalización (igual a 1) tenemos el 9,5 por ciento, mientras que en los datos norteamericanos alcanzaba el 9,8.

\footnotetext{
${ }^{4}$ El desglose es el siguiente: 1, no deslocalizable; 2, deslocalizable con considerable dificultad; 3, mixta o neutral; 4, deslocalizable con algo de dificultad; 5 , deslocalizable con poca o nula dificultad.
} 


\subsubsection{Empleo}

Para el empleo se utilizarán los datos de la Encuesta de Población Activa entre 2011 y 2018. Los datos de la EPA contienen información de las ocupaciones con un nivel de desagregación de tres dígitos. Como se ha dicho antes, a esta desagregación se han unido los datos de los indicadores de riesgo de automatización estimados por Frey y Osborne (2017) y del riesgo de deslocalización de Blinder y Krueger (2013), utilizando la conversión oficial desde la clasificación ocupacional americana a la Clasificación Internacional de Ocupaciones del año 2011. En caso de que una ocupación a tres dígitos incluyese varias categorías de las categorías originales (que tienen mayor desagregación) se ha atribuido la media de éstas a aquélla.

Estas ocupaciones están disponibles en la EPA para trabajadores por cuenta ajena y por cuenta propia, por lo que resulta especialmente adecuada para nuestro objetivo. Como el análisis se realizará desagregando por diferentes variables agregaremos todos los cuatrimestres del periodo mencionado, por lo que los resultados deben interpretarse como la media del periodo.

\subsection{Empleo total según nivel de riesgo de automatización y deslocalización}

Siguiendo Malo y Cueto (2019), el Cuadro 1 muestra las estimaciones realizadas para riesgo de automatización y deslocalización con los datos de la EPA en el periodo 2011-2018. Respecto al riesgo de automatización se han tomado los mismos límites que Frey y Osborne (2017) para definir los niveles alto, medio y bajo: alto por encima del 70 por ciento de riesgo de automatización; medio entre 30 y 70 por ciento; y bajo hasta el 30 por ciento. Como se puede observar, un 33,9 por ciento de los empleados estarían en puestos de trabajo con una probabilidad alta de automatización, un 40,8 con un nivel medio y un 25,3 con un nivel bajo. Esta distribución difiere de la que Frey y Osborne (2017) estimaron para el empleo norteamericano: 47, 19 y 33 por ciento, respectivamente. Así, en España el porcentaje de empleo bajo un riesgo alto de automatización es considerable, pues alcanza un tercio del empleo total, si bien en Estados Unidos se estimaba cercano a la mitad de todo el empleo. Por otro lado, en Estados Unidos había también una concentración importante en el riesgo bajo de automatización, mientras 
que en España es el riesgo medio el que agrupa a un mayor volumen de empleo (40,8 por ciento).

Dada la importante concentración en España de empleo en el nivel medio de riesgo de automatización, hemos añadido un umbral adicional en el 50 por ciento de riesgo de automatización para definir los niveles medio-bajo (riesgo entre 30 y 50 por ciento) y medio-alto (riesgo entre 50 y 70 por ciento). Se puede observar que un 27,9 por ciento estarían situados en el rango medio-alto. En definitiva, un 61,8 por ciento de los empleos tendrían un riesgo de automatización superior al 50 por ciento.

En términos del riesgo de deslocalización, en España un 66 por ciento del empleo total tendría un riesgo de deslocalización bajo, un 24,8 un riesgo medio y únicamente un 9,2 un riesgo alto. Recuérdese que, como se explicó con anterioridad, los riesgos están estimados de manera que riesgo bajo significa que el puesto de trabajo no es deslocalizable, riesgo medio que puede deslocalizarse con diferentes grados de dificultad, y riesgo alto que puede deslocalizarse sin dificultad. La distribución estimada para España es semejante a la que Blinder y Krueger (2013) encontraron para Estados Unidos: 68,4, 20,7 y 10,9 por ciento, respectivamente.

Como veremos a continuación, estos porcentajes varían de forma sustancial cuando se tiene en cuenta el tipo de empleo -por cuenta propia o ajena-de la persona.

Cuadro 1. Empleo total según estrato de riesgo de automatización y deslocalización (media 2011-2018). Fuente: elaboración propia a partir de EPA.

\begin{tabular}{|c|c|c|}
\hline & $\begin{array}{l}\text { Riesgo de automatización } \\
\text { Frey y Osborne (2017) }\end{array}$ & $\begin{array}{l}\text { Riesgo de deslocalización } \\
\text { Blinder y Krueger (2013) }\end{array}$ \\
\hline Bajo & 25,3 & 66,0 \\
\hline Medio & $\begin{array}{l}\text { Med - bajo } 12,9 \\
\text { Med - alto 27,9 }\end{array}$ & 24.8 \\
\hline Alto & 33,9 & 9,2 \\
\hline
\end{tabular}

\subsection{Empleo autónomo y riesgo de automatización}

En el Gráfico 1 se ha representado la distribución del empleo en función del riesgo de automatización, teniendo en cuenta de si se trata de empleo asalariado o autónomo (sin o con asalariados). Así, un 35,9 por ciento de empleo asalariado tiene un riesgo alto de automatización, mientras que, en el caso del empleo autónomo, dicho porcentaje se reduce 
hasta el 23,3 por ciento. En cambio, se sitúa en un riesgo bajo un 21,9 por ciento del empleo asalariado y un 42,4 del empleo autónomo. En las categorías de riesgo medio, los porcentajes son más similares en ambos tipos de empleo. Por tanto, se observa que el riesgo de automatización es más elevado para los ocupados por cuenta ajena que para los ocupados por cuenta ajena.

Gráfico 1. Distribución del empleo según niveles de riesgo de automatización, por tipo de empleo (media 2011-2018). Fuente: elaboración propia a partir de EPA.

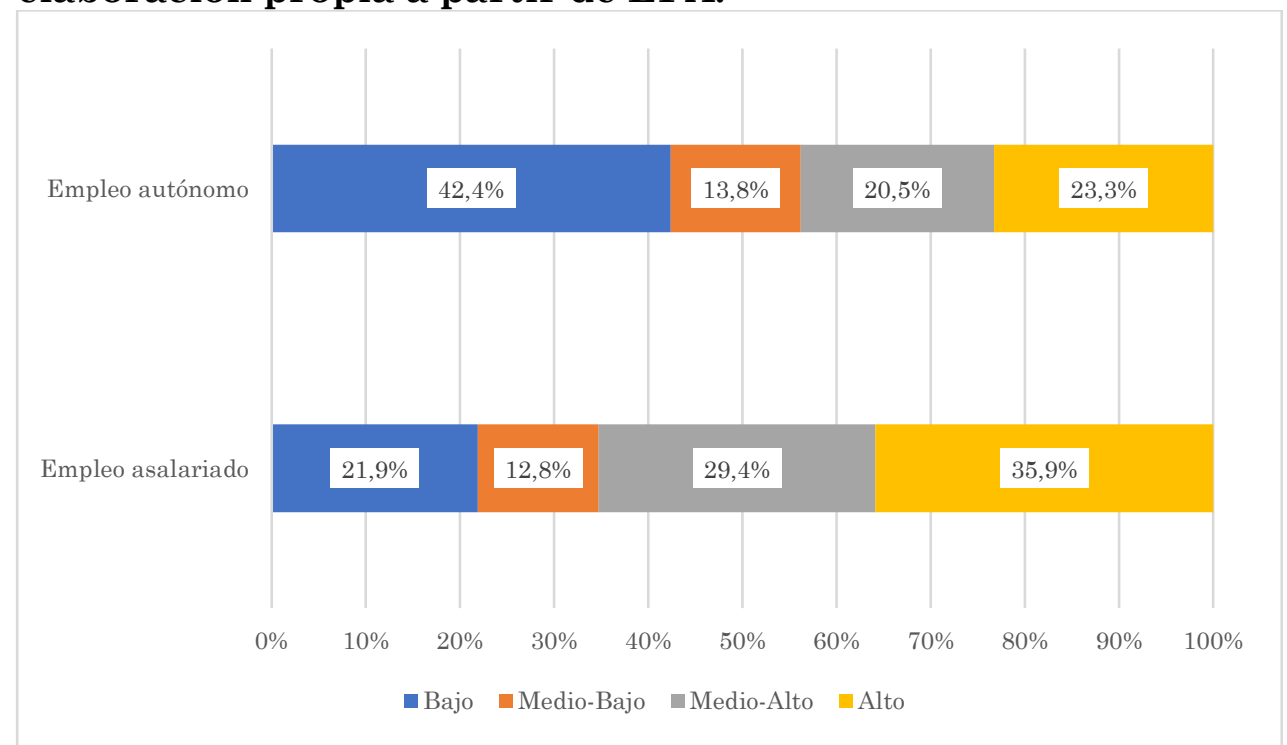

Nota: Autónomo recoge tanto sin como con asalariados.

Esta pauta de mayor concentración de los autónomos en el nivel más bajo de riesgo se ve igualmente reflejada cuando consideramos distintas características de los trabajadores (Cuadro 2). Por género, la concentración en el nivel de bajo riesgo para las mujeres autónomas es mayor (50,7 por ciento) que para los hombres autónomos (38,3 por ciento), mientras que para mujeres y hombres asalariados es prácticamente la misma (21,9 y 21,8 por ciento, respectivamente). Respecto al nivel educativo, las personas con bajo nivel de estudios tienen mayores riesgos que aquellas con estudios universitarios sea cual sea su nivel de empleo. Ahora bien, de nuevo la concentración en el nivel de bajo riesgo de automatización es mucho mayor entre los autónomos que entre los asalariados, llegando al 75,1 por ciento en el caso de los autónomos con nivel estudios universitarios, frente al 57,8 de los asalariados con el 
mismo nivel de estudios. En cuanto al nivel alto de riesgo de automatización, para los dos tipos de empleo hay más empleo concentrado en este nivel cuanto más bajo es el nivel de estudios. En los extremos, quienes tienen estudios obligatorios alcanzan un 35,3 por ciento entre los autónomos y un 49,7 entre los asalariados, mientras que quienes tienen estudios universitarios suponen un 6,1 por ciento entre los autónomos y un 14,9 entre los asalariados. Siguiendo los resultados que en Malo y Cueto (2019) se obtenían para el empleo total, un mayor nivel de estudios supone una mayor protección frente al riesgo de automatización. En todo caso, el Cuadro 2 muestra que esta pauta se une a la de menor riesgo de automatización de los autónomos frente a los asalariados, a lo que se añade una concentración en el bajo nivel de riesgo de automatización para las trabajadoras autónomas.

Cuadro 2. Distribución del empleo según nivel de riesgo de automatización, por tipo de empleo, género y nivel educativo (media 2011-2018). Fuente: elaboración propia a partir de EPA.

\begin{tabular}{|c|c|c|c|c|c|}
\hline & \multicolumn{5}{|c|}{ Asalariado } \\
\hline & Bajo & Medio-bajo & Medio - Alto & Alto & Total \\
\hline Total & 21,9 & 12,8 & 29,4 & 35,9 & 100 \\
\hline Hombre & 21,8 & 13,1 & 27,2 & 37,8 & 100 \\
\hline Mujer & 21,9 & 12,5 & 31,8 & 33,8 & 100 \\
\hline Estudios: & & & & & 100 \\
\hline Obligatorios & 4,7 & 10,9 & 34,7 & 49,7 & 100 \\
\hline FP Grado Medio & 8,1 & 21,9 & 30,3 & 39,7 & 100 \\
\hline Secundaria no obligatoria & 11,8 & 13,5 & 33,1 & 41,7 & 100 \\
\hline FP Grado Superior & 13,5 & 16,8 & 35,9 & 33,9 & 100 \\
\hline \multirow[t]{3}{*}{ Universitarios } & 57,8 & 9,8 & 17,5 & 14,9 & 100 \\
\hline & \multicolumn{5}{|c|}{ Autónomo } \\
\hline & Bajo & Medio-bajo & Medio - Alto & Alto & Total \\
\hline Total & 42,4 & 13,8 & 20,5 & 23,3 & 100 \\
\hline Hombre & 38,3 & 13,0 & 25,0 & 23,7 & 100 \\
\hline Mujer & 50,7 & 15,4 & 11,5 & 22,4 & 100 \\
\hline Estudios: & & & & & 100 \\
\hline Obligatorios & 27,4 & 11,1 & 26,2 & 35,3 & 100 \\
\hline FP Grado Medio & 29,5 & 23,7 & 23,3 & 23,5 & 100 \\
\hline Secundaria no obligatoria & 42,0 & 16,4 & 20,0 & 21,6 & 100 \\
\hline FP Grado Superior & 37,5 & 21,4 & 24,1 & 17,0 & 100 \\
\hline Universitarios & 75,1 & 10,2 & 8,7 & 6,1 & 100 \\
\hline
\end{tabular}

Nota: Autónomo recoge tanto sin como con asalariados.

Una cuestión que suele estar implícita en muchos debates sobre el empleo autónomo es si éstos son más bien trabajadores que no tienen empresario o si se trata de un empresario que no tiene trabajadores. Por ello, en el Cuadro 3 realizamos el mismo ejercicio que en el cuadro anterior, pero comparando empleadores y trabajadores autónomos sin asalariados. Se aprecia que estando ambos grupos mucho más concentrados en niveles bajos de riesgo que los asalariados que veíamos antes en el Cuadro 2, los empresarios lo están aún más que los 
trabajadores autónomos sin asalariados; así, tendrían un bajo nivel de riesgo de automatización el 55,1 por ciento de los empleadores y un 36,6 de los trabajadores por cuenta propia. Es más, la mejor situación de los empleadores se repite para cada una de las características consideradas, tanto en términos de género como de nivel educativo. De nuevo, se aprecia que el porcentaje de los que tienen bajo riesgo de automatización es creciente con el nivel educativo, con mayores valores para los empresarios que para los autónomos sin asalariados. Así pues, los datos podrían indicar que lo que hace menos susceptible a la automatización al empleo por cuenta propia en comparación con el empleo por cuenta ajena, se encuentra aún más presente entre los empresarios que entre los autónomos sin asalariados.

Cuadro 3. Distribución del empleo según nivel de riesgo de automatización, por tipo de empleo por cuenta propia, género y nivel educativo (media 2011-2018) Fuente: elaboración propia a partir de EPA.

\begin{tabular}{lccccc}
\hline & \multicolumn{5}{c}{ Empleador } \\
\hline & Bajo & Medio -bajo & Medio - Alto & Alto & Total \\
Total & 55,1 & 10,4 & 13,8 & 20,7 & 100 \\
\hline Hombre & 53,8 & 9,2 & 16,1 & 20,9 & 100 \\
Mujer & 58,2 & 13,3 & 8,4 & 20,2 & 100 \\
Estudios: & & & & \\
Obligatorios & 41,3 & 8,6 & 18,5 & 31,6 & 100 \\
FP Grado Medio & 40,2 & 21,7 & 15,2 & 23,0 & 100 \\
Secundaria no obligatoria & 58,6 & 9,7 & 12,0 & 19,6 & 100 \\
FP Grado Superior & 49,3 & 16,7 & 17,0 & 16,9 & 100 \\
Universitarios & 80,3 & 7,2 & 6,1 & 6,3 & 100 \\
\hline & Trabajador por cuenta propia sin empleados \\
\hline & Bajo & Medio-bajo & Medio - Alto & Alto & Total \\
\hline Total & 36,6 & 15,3 & 23,6 & 24,4 & 100 \\
Hombre & 30,9 & 14,9 & 29,2 & 25,0 & 100 \\
Mujer & 47,7 & 16,2 & 12,8 & 23,3 & 100 \\
Estudios: & \multicolumn{4}{c}{} \\
Obligatorios & 21,9 & 12,1 & 29,3 & 36,8 & 100 \\
FP Grado Medio & 25,0 & 24,6 & 26,7 & 23,7 & 100 \\
Secundaria no obligatoria & 32,8 & 20,1 & 24,4 & 22,7 & 100 \\
FP Grado Superior & 31,6 & 23,7 & 27,6 & 17,1 & 100 \\
Universitarios & 72,5 & 11,6 & 9,9 & 6,0 & 100 \\
\hline
\end{tabular}

Tanto el Cuadro 2 como el Cuadro 3 parecen apuntar que las características estarían detrás de una pauta general que afecta tanto a trabajadores por cuenta ajena como por cuenta propia y a los distintos tipos de estos últimos. Por ello, pasamos a explorar si la concentración de 
empleo asalariado y autónomo se da por igual en diferentes sectores de actividad $^{5}$.

El Gráfico 2 muestra el porcentaje de empleo que se concentra en el nivel de bajo riesgo de automatización, por tipo de empleo y por sector de actividad. Resulta muy llamativo que la rama de actividad en que trabaja la persona conduce a diferencias muy relevantes en función del tipo de empleo. Sólo en cinco categorías, el porcentaje de empleos con riesgo bajo de automatización es mayor para los asalariados que para los autónomos ${ }^{6}$. Cabe destacar el caso de educación, para el que el porcentaje de empleos con bajo riesgo de automatización es relativamente elevado y, a la vez, casi igual para asalariados y autónomos.

En el resto de ramas de actividad, las diferencias son, en general, sustanciales, apuntando que, aunque trabajen en la misma actividad, la naturaleza de las tareas realizadas por asalariados y autónomos serían diferentes. Las ramas con menores riesgos de automatización corresponden, en general, al sector servicios. Se trata de actividades realizadas por profesionales con alto nivel de cualificación, tales como sanidad, investigación y desarrollo o servicios a empresas.

El Gráfico 3 muestra el caso opuesto, es decir, el porcentaje de empleo con riesgo alto de automatización, de nuevo por tipo de empleo y rama de actividad. Aquí, los mayores porcentajes se sitúan en las actividades relacionadas con los sectores primario y secundario, y también con algunos servicios, como es la hostelería. Es importante señalar, que, entre las que se encuentran en los primeros puestos, las diferencias entre empleo autónomo y asalariado son pequeñas. En el resto, se observa una gran disparidad entre ambos tipos de empleo, si bien con porcentajes usualmente superiores para el empleo asalariado, a diferencia de lo que sucedía en el gráfico anterior y en consonancia con lo que apuntaban los cuadros anteriores (mayor concentración en riesgo alto de automatización de los asalariados).

\footnotetext{
${ }^{5}$ En todos los gráficos, los sectores de actividad se corresponden con la desagregación a dos dígitos de la actual Clasificación Nacional de Actividades Económicas.

${ }^{6}$ Estas actividades son: servicios financieros no seguros y fondos de pensiones; otras industrias manufactureras; seguros y fondos de pensiones (no incluye los relacionados con la seguridad social obligatoria); actividades de apoyo a las industrias extractivas; y pesca y acuicultura.
} 


\section{Gráfico 2. Porcentajes de empleo con riesgo bajo de automatización, por tipo de empleo y rama de actividad. Fuente: elaboración propia a partir de EPA.}

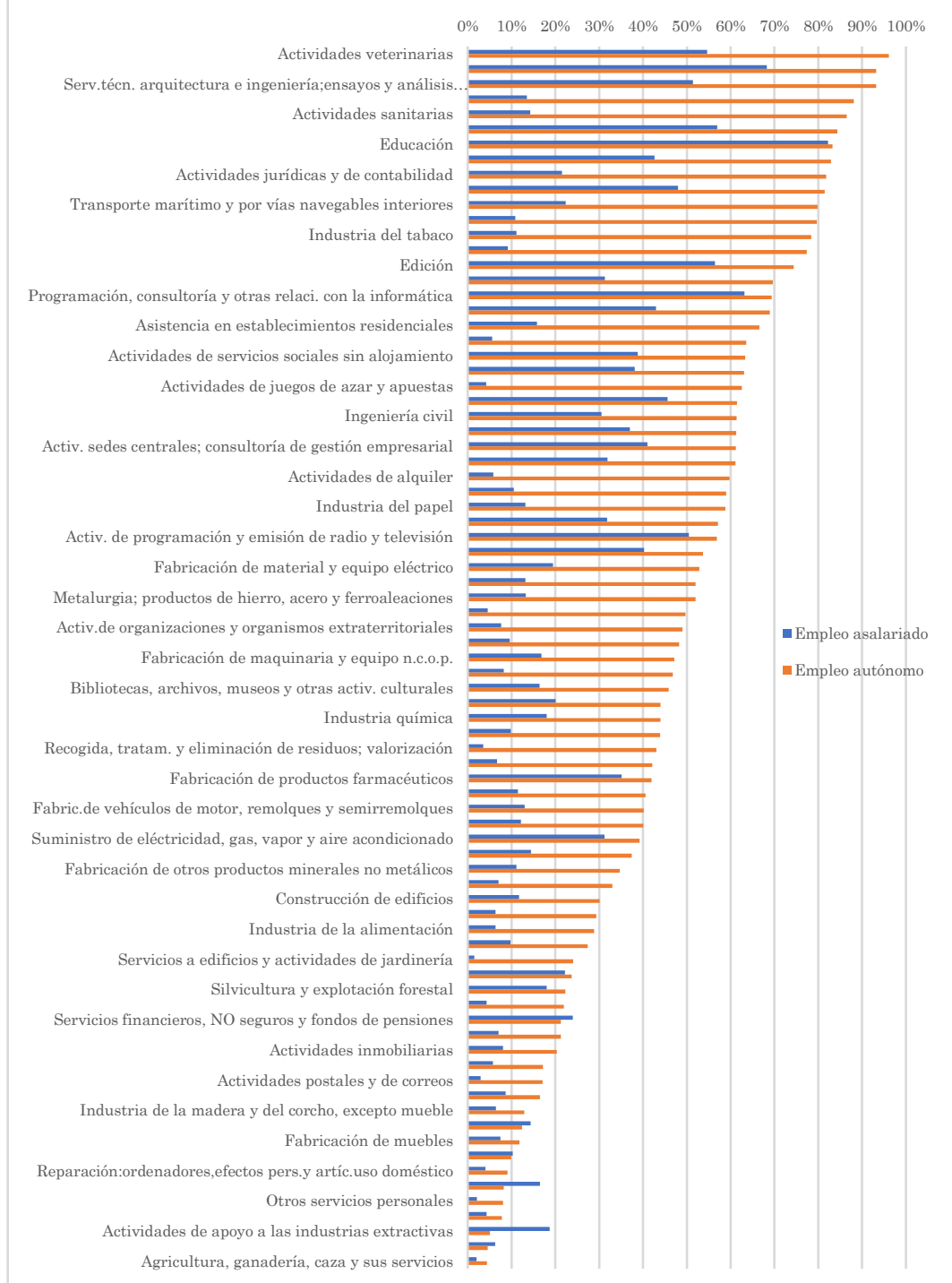

Nota: Autónomo recoge tanto sin como con asalariados. 
Gráfico 3. Porcentajes de empleo con riesgo alto de automatización, por tipo de empleo y rama de actividad. Fuente: elaboración propia a partir de EPA.

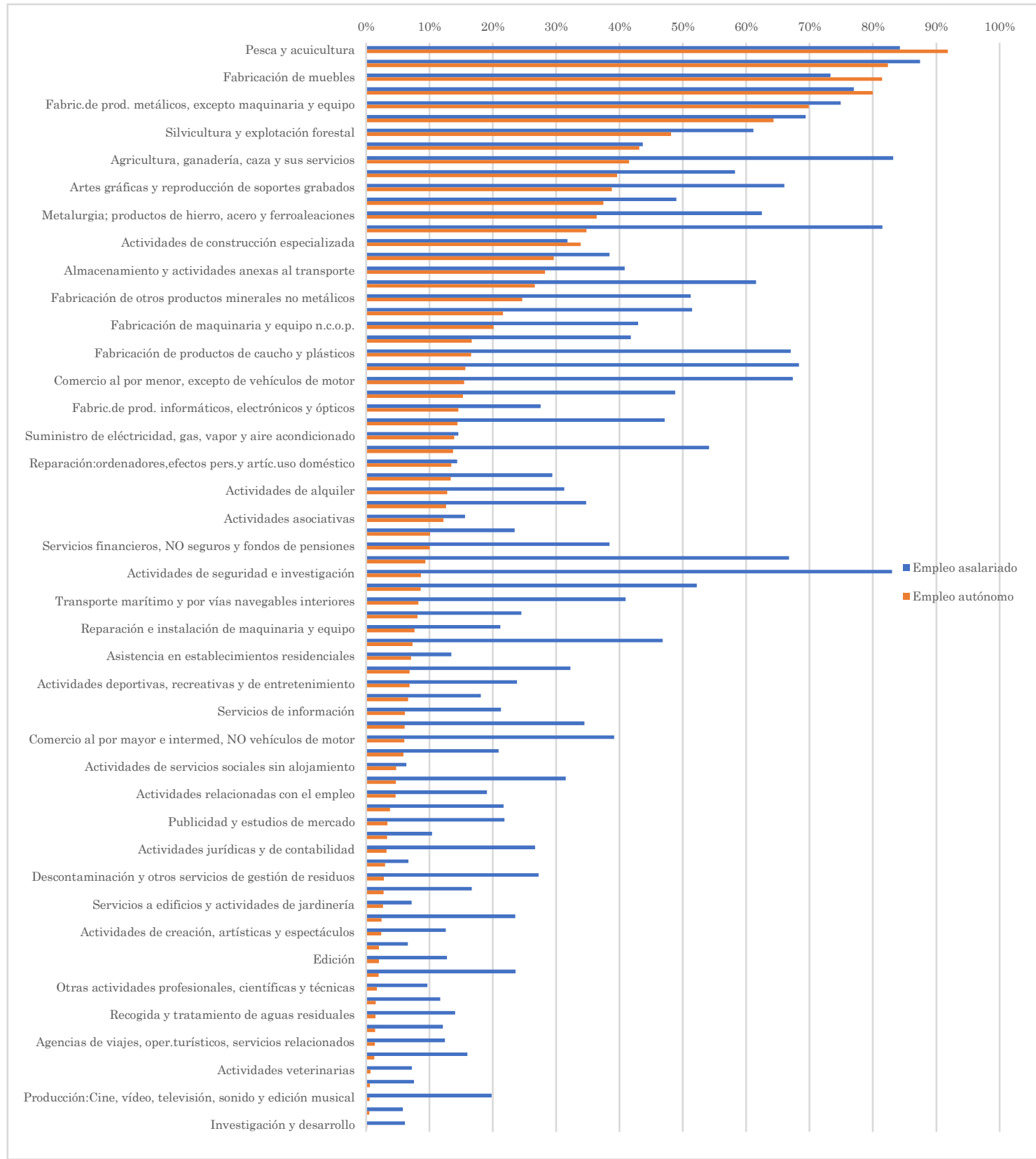

Nota: Autónomo recoge tanto sin como con asalariados. 
En definitiva, los datos parecen apuntar que las diferencias en los riesgos de automatización por tipo de empleo no radicarían en que los autónomos tienen características diferentes de los asalariados, sino más bien en que se concentran en actividades diferentes. Esto último sugiere que el contenido de las ocupaciones (en términos de tareas) no sería exactamente el mismo en el empleo asalariado y en el autónomo, algo que, desafortunadamente, no podemos explorar con los datos disponibles.

\subsection{Empleo autónomo y riesgo de deslocalización}

Si atendemos al riesgo de deslocalización, los resultados están en la línea de los obtenidos para el riesgo de automatización. De esta forma, tal y como se puede observar en el Gráfico 4, la proporción de puestos con riesgo igual a 1 es de un 10,4 por ciento en el caso del empleo asalariado, mientras que se reduce a la mitad en el caso del empleo autónomo. En cambio, el porcentaje de empleos con riesgo igual a 0 es diez puntos superior en el caso del empleo autónomo (72,2 por ciento) en comparación con el empleo asalariado (63,4 por ciento).

Gráfico 4. Distribución del empleo por nivel de riesgo de deslocalización y tipo de empleo (media 2011-2018). Fuente: elaboración propia a partir de EPA.

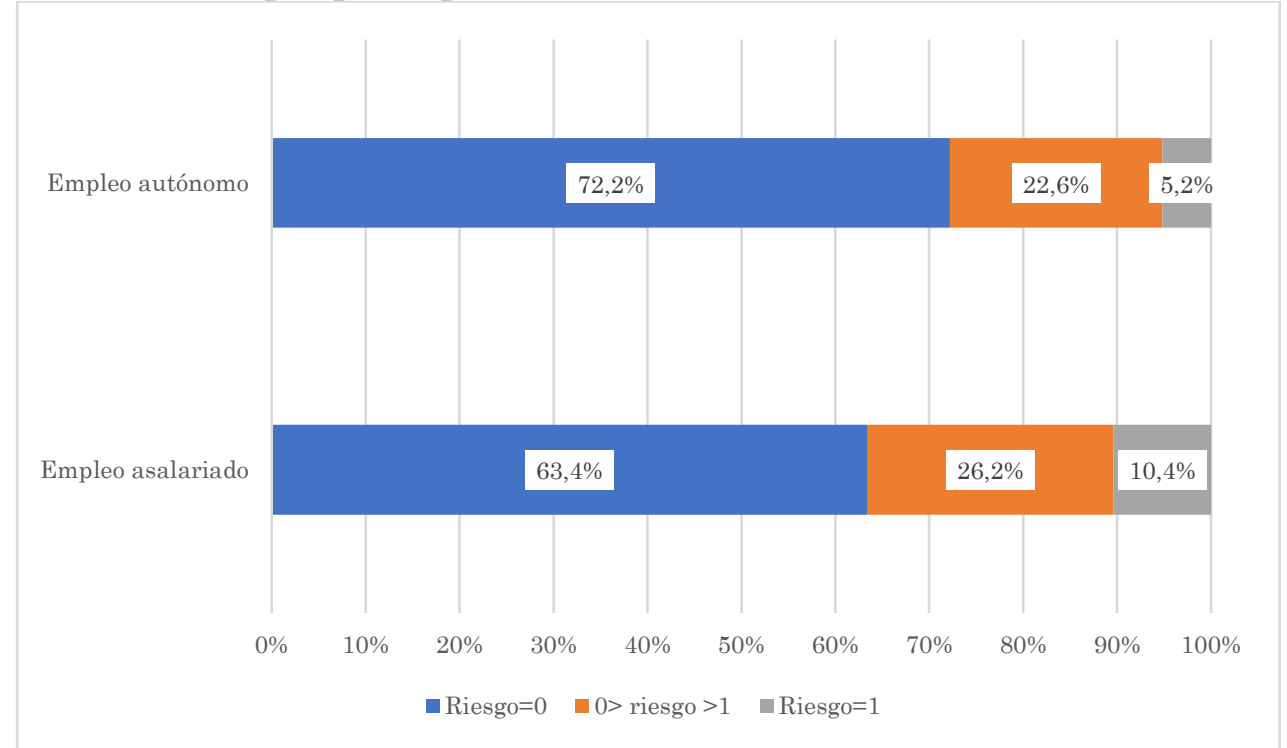

Nota: Autónomo recoge tanto sin como con asalariados. 
Cuando se diferencia en función del género y del nivel educativo, se obtienen los resultados del Cuadro 4. En todos los casos, se observa que la concentración en el grupo de alto riesgo de deslocalización es menor para el empleo autónomo. Teniendo en cuenta el género, la distribución del empleo por niveles de riesgo de deslocalización es similar para hombres y mujeres ocupados por cuenta propia; en cambio, los hombres asalariados están más concentrados en el grupo de mayor riesgo que las mujeres asalariadas. En términos de nivel educativo, los porcentajes de empleo en el riesgo de deslocalización más alto es menor entre las personas con menores niveles de cualificación, tanto para empleo asalariado como autónomo. Ahora bien, las diferencias para secundaria no obligatoria, formación profesional superior y universidad son reducidas, especialmente entre los autónomos.

Cuadro 4. Distribución del empleo según nivel de riesgo de deslocalización, por tipo de empleo, género y nivel educativo (media 2011-2018). Fuente: elaboración propia a partir de EPA.

\begin{tabular}{|c|c|c|c|c|}
\hline & \multicolumn{4}{|c|}{ Asalariado } \\
\hline & riesgo $=0$ & $0>$ riesgo $>1$ & riesgo $=1$ & Total \\
\hline Total & 63,4 & 26,2 & 10,4 & 100 \\
\hline Hombre & 59,6 & 27,5 & 12,9 & 100 \\
\hline Mujer & 67,4 & 24,8 & 7,8 & 100 \\
\hline Estudios: & & & & 100 \\
\hline Obligatorios & 78,7 & 12,9 & 8,4 & 100 \\
\hline FP Grado Medio & 73,4 & 17,5 & 9,1 & 100 \\
\hline Secundaria no obligatoria & 65,0 & 24,5 & 10,5 & 100 \\
\hline FP Grado Superior & 54,9 & 31,2 & 13,8 & 100 \\
\hline \multirow[t]{3}{*}{ Universitarios } & 42,7 & 45,3 & 12,0 & 100 \\
\hline & \multicolumn{4}{|c|}{ Autónomo } \\
\hline & riesgo $=0$ & $0>$ riesgo $>1$ & riesgo $=1$ & Total \\
\hline Total & 72,2 & 22,6 & 5,2 & 100 \\
\hline Hombre & 71,2 & 23,0 & 5,8 & 100 \\
\hline Mujer & 74,2 & 21,7 & 4,1 & 100 \\
\hline Estudios: & & & & 100 \\
\hline Obligatorios & 84,6 & 11,8 & 3,6 & 100 \\
\hline FP Grado Medio & 80,4 & 14,4 & 5,2 & 100 \\
\hline Secundaria no obligatoria & 70,2 & 22,9 & 6,9 & 100 \\
\hline FP Grado Superior & 67,8 & 25,6 & 6,6 & 100 \\
\hline Universitarios & 50,9 & 42,7 & 6,4 & 100 \\
\hline
\end{tabular}

Nota: Autónomo recoge tanto sin como con asalariados.

Respecto a las diferencias entre empleadores y autónomos sin trabajadores (Cuadro 5), la distribución del empleo por riesgo de deslocalización es similar para ambos grupos, a diferencia de lo que ocurría en el caso del riesgo de automatización examinado en la sección anterior. En cuanto a las diferencias observadas por género y nivel de estudios se mantiene lo ya observado en el Cuadro 4 en líneas generales, 
si bien los autónomos sin trabajadores están algo más concentrados tanto en el riesgo más bajo (con la excepción de las mujeres, quienes tienen secundaria no obligatoria y los universitarios) como en el riesgo más alto de deslocalización (sin excepciones). En todo caso, los mayores porcentajes de empleo concentrado en la más baja exposición al riesgo de deslocalización se dan entre los autónomos sin asalariados que tienen estudios obligatorios (87,3 por ciento) o formación profesional de grado medio ( 81,7 por ciento).

En definitiva, en líneas generales se aprecia la situación contraria al riesgo de automatización, pues en el caso de la deslocalización quienes soportan un menor riesgo parecen ser los autónomos sin asalariados con determinadas características. Ahora bien, las diferencias entre empleados y autónomos sin asalariados no parecen tan marcadas como sucedía en el caso de riesgo de automatización.

Cuadro 5. Distribución del empleo según nivel de riesgo de deslocalización, tipo de empleo por cuenta propia, género y nivel educativo (media 2011-2018). Fuente: elaboración propia a partir de EPA.

\begin{tabular}{|c|c|c|c|c|}
\hline & \multicolumn{4}{|c|}{ Empleador } \\
\hline & Riesgo $=0$ & 0>Riesgo $>1$ & Riesgo $=1$ & Total \\
\hline Total & 71,4 & 25,1 & 3,5 & 100 \\
\hline Hombre & 68,9 & 27,3 & 3,8 & 100 \\
\hline Mujer & 77,2 & 20,1 & 2,7 & 100 \\
\hline \multicolumn{5}{|l|}{ Estudios: } \\
\hline Obligatorios & 78,0 & 19,5 & 2,6 & 100 \\
\hline FP Grado Medio & 77,1 & 18,9 & 4,0 & 100 \\
\hline Secundaria no obligatoria & 71,7 & 24,8 & 3,5 & 100 \\
\hline FP Grado Superior & 67,5 & 28,9 & 3,7 & 100 \\
\hline \multirow[t]{3}{*}{ Universitarios } & 61,5 & 33,9 & 4,5 & 100 \\
\hline & \multicolumn{4}{|c|}{ Trabajador por cuenta propia sin empleados } \\
\hline & Riesgo $=0$ & 0>Riesgo $>1$ & Riesgo $=1$ & Total \\
\hline Total & 72,5 & 21,5 & 6,0 & 100 \\
\hline Hombre & 72,3 & 21,0 & 6,7 & 100 \\
\hline Mujer & 72,9 & 22,4 & 4,6 & 100 \\
\hline \multicolumn{5}{|l|}{ Estudios: } \\
\hline Obligatorios & 87,3 & 8,7 & 4,0 & 100 \\
\hline FP Grado Medio & 81,7 & 12,6 & 5,7 & 100 \\
\hline Secundaria no obligatoria & 69,3 & 21,9 & 8,8 & 100 \\
\hline FP Grado Superior & 68,0 & 23,9 & 8,1 & 100 \\
\hline Universitarios & 45,6 & 47,1 & 7,3 & 100 \\
\hline
\end{tabular}

El análisis de los dos cuadros anteriores indicaría que, en el caso del riesgo de deslocalización, las diferencias en características podrían tener algo más de importancia que en el caso de la automatización, pero únicamente para la diferenciación entre tipos de empleo por cuenta 
propia. Por ello, al igual que en la sección anterior procedemos a explorar las diferencias en cuanto a porcentajes de empleo en los niveles bajo (Gráfico 5) y alto (Gráfico 6) de riesgo de deslocalización por rama de actividad.

En cuanto al riesgo bajo de deslocalización (Gráfico 5), para la misma rama de actividad los porcentajes de empleo son más bien diferentes y, aunque hay una cierta mayor concentración en este nivel bajo de riesgo de deslocalización entre los autónomos, también nos encontramos con un amplio número de ramas de actividad en las que los porcentajes de empleo son mayores entre los asalariados. Así pues, la pauta que se observaba para el riesgo de automatización, en el sentido de que el empleo por cuenta propia tuviera menores riesgos para casi todas las actividades, no se aprecia en este caso.

Las ramas de actividad en las que se concentra más empleo con bajo riesgo de deslocalización son aquellas en las que existe un mayor contacto con los clientes, es decir, aquellas en las que la presencialidad es un factor clave de la prestación del servicio. Así, se sitúan en los primeros puestos, servicios personales, hostelería o comercio, entre otros. En cambio, tienen un riesgo alto de deslocalización las vinculadas a la fabricación de productos, ramas fundamentalmente de tipo industrial.

En cuanto al riesgo alto de deslocalización (Gráfico 6), se aprecia que afecta más al empleo asalariado en casi todas las ramas de actividad que al empleo autónomo. Las excepciones que afectan a mayores porcentajes de empleo dentro de la actividad son: fabricación de productos farmacéuticos y fabricación de muebles. Se trata, pues, de actividades cuyo riesgo de deslocalización más que a una ocupación en particular podrían afectar a la empresa entera, algo que tendría impacto tanto sobre el empleo asalariado como sobre el autónomo.

En definitiva, en el caso del riesgo de deslocalización no parece tan claro que las distintas características de los trabajadores afecten de manera parecida a asalariados y autónomos. Aunque los datos apuntan, en ocasiones, que es la diferente relación de empleo la que marca una diferencia clave para entender la distribución del empleo por niveles de exposición al riesgo de deslocalización, no hay que descartar que las características de los sectores (que afectarían por igual a asalariados y autónomos) sean también relevantes. 


\section{Gráfico 5. Porcentaje de empleo con riesgo bajo de deslocalización, por tipo de empleo y rama de actividad. Fuente: elaboración propia a partir de EPA.}

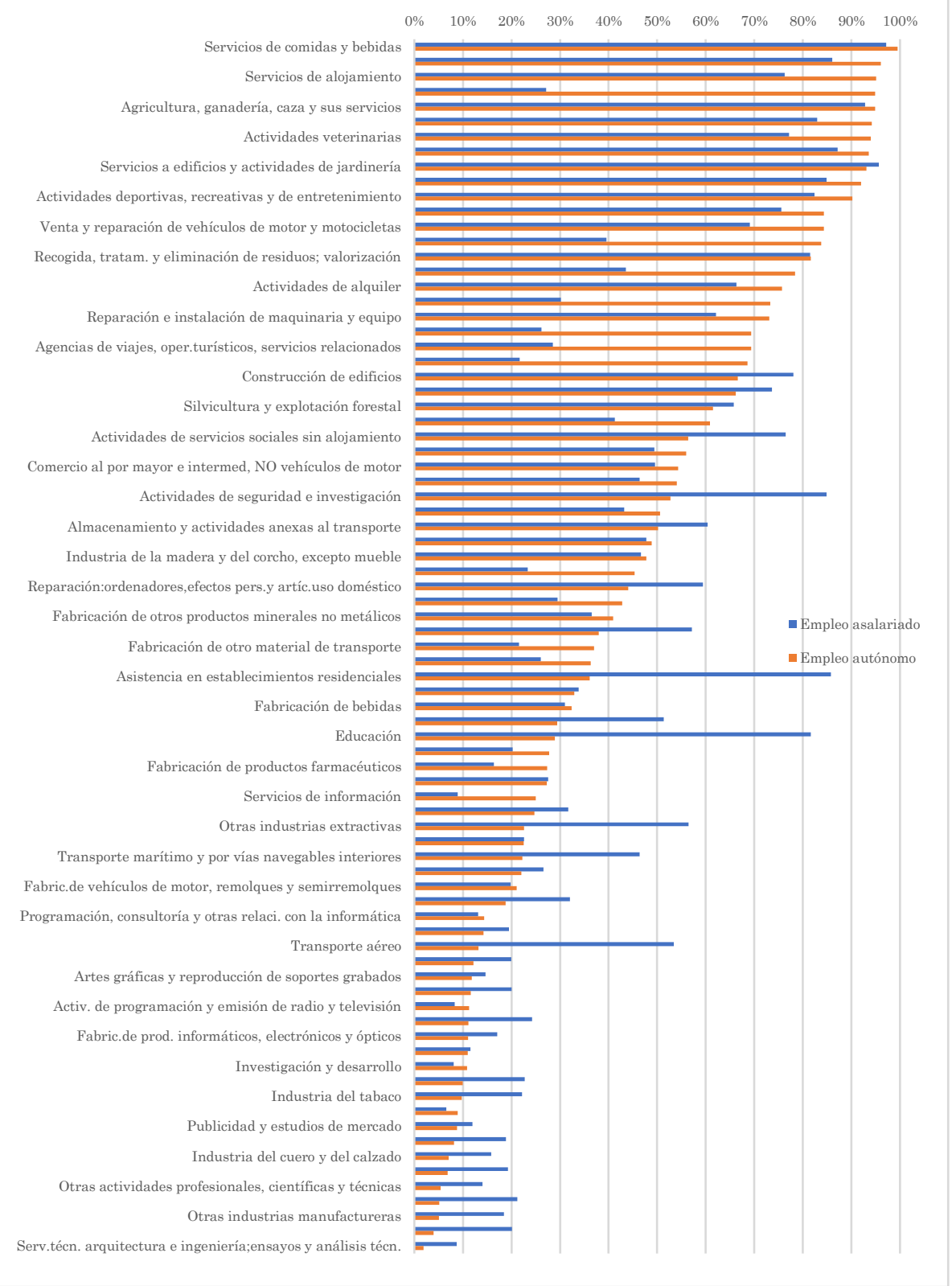

Nota: Autónomo recoge tanto sin como con asalariados. 
Gráfico 6. Porcentaje de empleo con riesgo alto de deslocalización, por tipo de empleo y rama de actividad. Fuente: elaboración propia a partir de EPA.

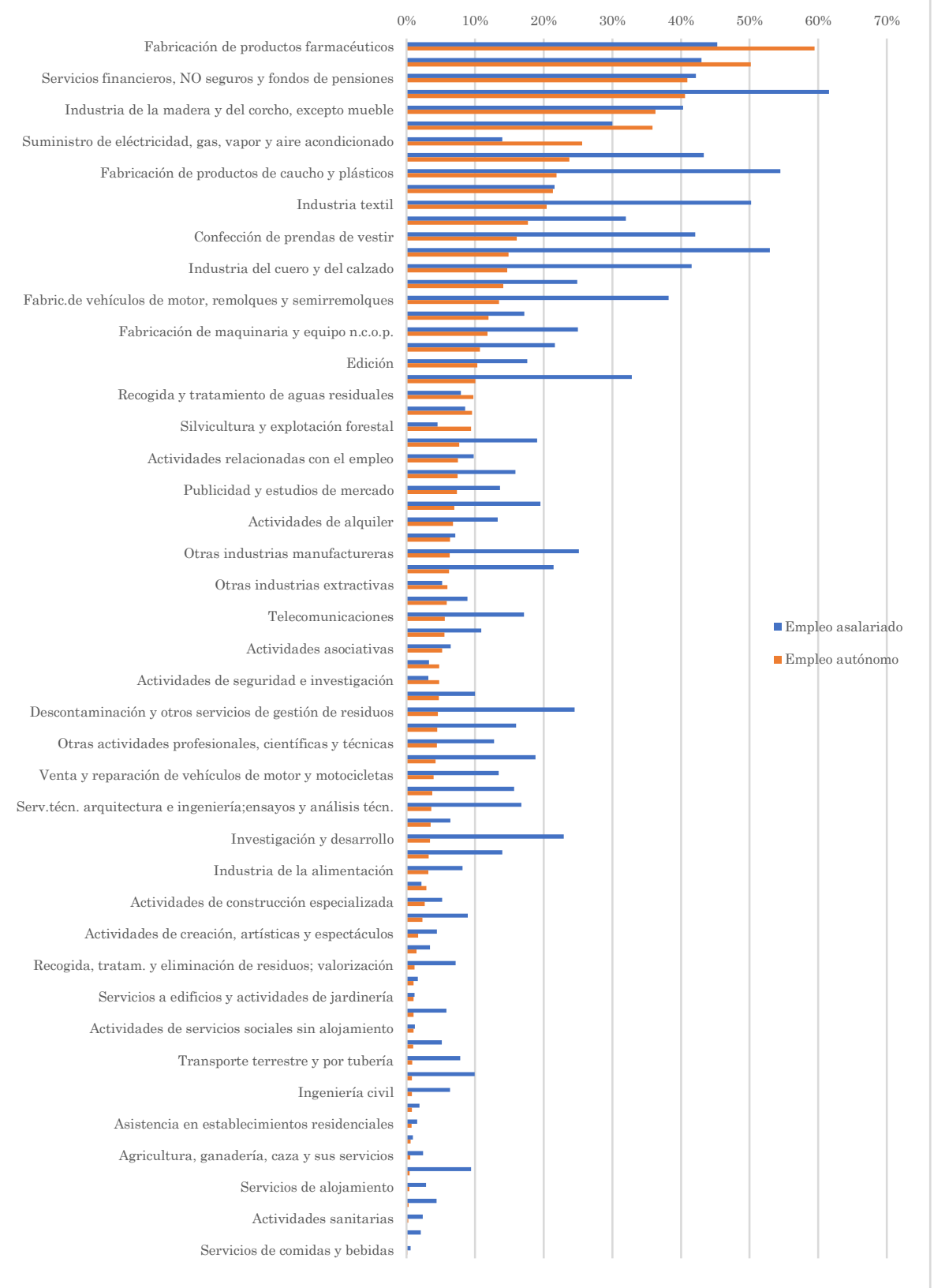

Nota: Autónomo recoge tanto sin como con asalariados. 


\section{Discusión de conjunto y conclusiones}

Los resultados muestran diferencias relevantes entre empleo por cuenta propia y ajena en los riesgos de automatización y deslocalización. En cuanto al riesgo de automatización, los autónomos se concentran mucho más en ocupaciones con bajo riesgo de ser automatizadas. Dentro del empleo por cuenta propia, los datos muestran también diferencias en este segundo grupo. Así, es importante distinguir entre empleadores y ocupados por cuenta propia sin empleados, pues la concentración en ocupaciones de bajo riesgo de automatización es aún más acusada entre los empleadores.

Por otro lado, la diferenciación por características como el género y el nivel educativo muestran que, para los autónomos, se dan patrones de riesgo de automatización similares a los del empleo asalariado, siempre guardando la anterior regularidad según la cual hay más concentración de los autónomos en ocupaciones de bajo riesgo de automatización. Esto indicaría que, posiblemente, hay diferencias entre las mismas ocupaciones de asalariados y autónomos que no están siendo consideradas por la metodología que estima los riesgos de automatización por ocupaciones.

El estudio por actividades nos muestra también resultados que se pueden analizar en línea con esta interpretación. Así pues, a la hora de examinar los desafíos que el actual cambio técnico puede traer para el empleo autónomo (y para el empleo por cuenta propia en general) convendría avanzar más allá de las actuales metodologías que estiman riesgos de automatización por ocupaciones (Frey y Osborne, 2017; Arntz et al, 2016; Nedelkoska y Quintini, 2018; Pouliakas, 2018) y que, implícitamente, están desarrolladas asumiendo que la relación laboral es asalariada y se presta dentro de una empresa junto con otros trabajadores. Otros resultados como los de Sorgner (2017) mostrando que el cambio técnico puede favorecer transiciones de asalariados al autoempleo en ocupaciones con bajo riesgo de automatización podrían reforzar la representación dentro de los autónomos del porcentaje de quienes tienen un bajo riesgo de que sus trabajos se automaticen. Por tanto, se necesita futura investigación que permita distinguir cuál es la principal explicación de estas grandes diferencias en la baja exposición de los empleados por cuenta propia al riesgo de automatización en comparación con los asalariados.

Por lo que respecta al riesgo de deslocalización, los resultados no son tan claros. Sí es cierto que, en agregado, el empleo autónomo tiene una mayor (menor) concentración en ocupaciones de bajo (alto) riesgo de 
deslocalización que el empleo asalariado. Es la desagregación por características la que marca la diferencia respecto del riesgo de automatización. Por un lado, las diferencias dentro del empleo por cuenta propia entre empleadores y autónomos sin asalariados no son tan intensas como anteriormente. Por otro lado, las diferencias en características parecen ser relevantes en algún grado para entender la menor exposición al riesgo de deslocalización de los trabajadores autónomos. La escasez de literatura previa, hasta donde nosotros sabemos, nos impide aventurar más como en el caso del riesgo de automatización. Esto muestra de forma clara que la muy frecuente asimilación de los efectos de la deslocalización a los de la automatización y a que las políticas preventivas irían en la misma dirección puede ser un planteamiento erróneo. Se necesita, pues, abrir nuevas rutas de investigación que permitan entender la exposición a las transformaciones del trabajo autónomo frente a los efectos de la globalización, en particular al riesgo de deslocalización.

Finalmente, cabe señalar que, con los datos existentes, no es posible estimar adecuadamente los riesgos de automatización y deslocalización para un colectivo de gran interés como son los autónomos dependientes o falsos autónomos. En este caso, si las tareas realizadas son similares a las de los asalariados, no podríamos decir que el empleo autónomo, por denominarse de esta forma, tiene un menor riesgo de automatización, sino que sería más parecido al del empleo asalariado. Más allá del empleo a través de plataformas digitales, el empleo transformado por los cambios tecnológicos tiene todavía muchos componentes desconocidos y no sabemos aún hasta qué punto las novedades realmente están dando lugar a un nuevo tipo de empleo por cuenta propia o no.

\section{Referencias bibliografícas}

Anderson, R.G. y Gascon, C.S. (2008): "Offshoring, Economic Insecurity, and the Demand for Social Insurance”, working paper 2008-003A, Research Division, Federal Reserve Bank of St. Louis, Working Paper Series.

Arntz, M., Gregory, T. y Zierahn, U. (2016): "The risk of automation for jobs in OECD countries: A comparative analysis", OECD Social, Employment and Migration working papers, $\mathrm{N}^{\circ}$. 189, OECD, Paris. 
Arum R. y Müller, W. (2009): The Reemergence of Self-Employment: A Comparative Study of Self-Employment Dynamics and Social Inequality. pp. 1466. Princeton University Press

Autor, D., Levy, F. y Murnane, R. (2003): "The skill content of recent technological change: An empirical exploration", Quarterly Journal of Economics, 188: 1279-1333.

Baldwin, R. (2019): The Globotics Upheaval. Globalization, Robotics, and the Future of Work. Oxford University Press.

Blinder, A.S. (2009): "How many US jobs might be offshorable?" World Economics 10 (2): 41-78.

Blinder, A.S. y Krueger, A.B. (2013): "Alternative Measures of Offshorability: A Survey Approach”, Journal of Labor Economics, 31(2), S97-S128.

Bögenhold, D., Klinglmair, R. y Kandutsch, F. (2018): "Self-employment on the way in a digital economy: A variety of shades of grey". IFS Discussion Papers, Vol. 2018, No. 01 (28 February 2018): pp. 1-25.

Cabeza Pereiro, J. (2008): El estatuto del trabajador autónomo español. Revista Internacional Del Trabajo, 127(1), 99-108.

Eurofound (2018): Employment and working conditions of selected types of platform work. Publications Office of the European Union, Luxembourg.

De Groen, W.P. y Maselli, I. (2016): "The impact of the Collaborative Economy on the Labour Market", CEPS special report, Center for European Policy Studies, Brussels.

De Stefano, V. (2016): "The rise of the just-in-time workforce: On-demand work, crowdwork and labour protection in the gig economy", Conditions of Work and Employment Series No. 71, INWORK, Organización Internacional del Trabajo, Ginebra (Enlace: https://www.ilo.org/wcmsp5/groups/public/---ed_protect/--protrav/---travail/documents/publication/wcms_443267.pdf)

Domènech, R., García, J.R., Montáñez, M. y Neut, A. (2018): “Afectados por la revolución digital: El caso español”, Papeles de Economía Española, 156, 128 145 .

Eurofound (2018): Employment and working conditions of selected types of platform work. Oficina de publicaciones de la Unión Europea, Luxemburgo. Fernández-Macías, E., Bisello, M., Sarkar, S. y Torrejón, S. (2016): Methodology of the construction of task indices for the European Jobs Monitor. Eurofound. Enlace: https://www.eurofound.europa.eu/sites/default/files/ef1617en2.pdf 
Fernández Rodríguez, M.L. (2019): "Calificación jurídica de la relación que une a los prestadores de servicios con las plataformas digitales", capítulo 3 en M.L. Rodríguez Fernández (dir.), Plataformas digitales y mercado de trabajo, Ministerio de Trabajo, Madrid, págs. 57-89.

Frey, C.B. y Osborne, M.A. (2017): "The future of employment: How susceptible are jobs to automation?" Technological Forecasting \& Social Change, 114, 254280.

Hidalgo, M.A. (2018): El empleo del futuro. Un análisis del impacto de las nuevas tecnologías en el mercado laboral. Ediciones Deusto, Barcelona.

Kässi, O. y Lehdonvirta, V. (2018): "Online Labour Index: Measuring the Online Gig Economy for Policy and Research". Technological Forecasting and Social Change, 37: 241-248.

Kratz, L. y Krueger, A. (2016): "The Rise and Nature of Alternative Work Arrangements in the United States, 1995-2015”, NBER Working Paper 22667.

Malo, M.Á. (2017): "Nuevas formas de empleo: Del empleo atípico a las plataformas digitales", Papeles de Economía Española, 156: 146-158.

Malo, M.Á. y Cueto, B. (2019): "Do old and new labour market risks overlap? Automation, offshorability, and non-standard employment", MPRA working paper 95058 .

Muehlberger, U. (2007): Dependent Self-employment, Palgrave Macmillan.

Nedelkoska, L. y Quintini, G. (2018): “Automation, skills use and training,

OECD Social, Employment and Migration working papers, N. 202, OECD, Paris.

OCDE (2019): Employment outlook 2019, París.

OIT (2018): Digital labour platforms and the future of work: Towards decent work in the online world. International Labour Organization, Geneva (Switzerland).

Pouliakas, K. (2018): "Determinants of automation risk in the EU labour market: A skills-needs approach”, IZA discussion papers, No .11829.

Sorgner, A. (2017): "The Automation of Jobs: A Threat for Employment or a Source of New Entrepreneurial Opportunities". Foresight and STI Governance, 11(3): 37-48. 
Torrejón, S. (2018): “Ocupaciones y trabajadores vulnerables: Los cambios del lado de la demanda de empleo y sus implicaciones desde el punto vista de la desigualdad y el género", Revista de Economía Laboral 14(2): 137-168.

Weill, D. (2014): The fissured workplace, Harvard University Press, Cambridge (US). 